\title{
ADAPTIVE FINITE ELEMENT RELAXATION SCHEMES FOR HYPERBOLIC CONSERVATION LAWS
}

\author{
Christos Arvanitis ${ }^{1,3}$, Theodoros Katsaounis $^{2,3}$ and Charalambos \\ MAKRIDAKIS ${ }^{1,3}$
}

\begin{abstract}
We propose and study semidiscrete and fully discrete finite element schemes based on appropriate relaxation models for systems of Hyperbolic Conservation Laws. These schemes are using piecewise polynomials of arbitrary degree and their consistency error is of high order. The methods are combined with an adaptive strategy that yields fine mesh in shock regions and coarser mesh in the smooth parts of the solution. The computational performance of these methods is demonstrated by considering scalar problems and the system of elastodynamics.
\end{abstract}

Mathematics Subject Classification. 35L65, 65M60, 65M50, 82C40.

Received: April 4, 2000. Revised: October 6, 2000.

\section{INTRODUCTION}

Most of the recent investigations towards designing efficient and robust numerical schemes for systems of Hyperbolic Conservation Laws

$$
\left\{\begin{array}{l}
\partial_{t} u+\sum_{i=1}^{N} \partial_{x_{i}} F_{i}(u)=0, \quad x \in \mathbb{R}^{N}, t>0, u(x) \in \mathbb{R}^{M} \\
u(x, 0)=u_{0}(x)
\end{array}\right.
$$

are based on finite difference or finite volume discretizations. The main difficulty, from the numerical point of view, is to choose the right regularization mechanism that can resolve with high accuracy and without spurious oscillations the shock regions, as well as the smooth parts of the solution. In this paper we propose finite element schemes for (1.1) in which the regularization mechanism is a combination of regularization by wave operators and of appropriate mesh refinement close to the regions of singularity. This can be done simply by applying a finite element discretization to the relaxation approximation of (1.1) and by selecting the mesh with the help of error indicators that can detect the location of the shock.

To motivate our discussion consider the scalar one dimensional problem

$$
\partial_{t} u+f(u)_{x}=0, \quad x \in \mathbb{R}, t>0, u(x) \in \mathbb{R} .
$$

\footnotetext{
Keywords and phrases. Conservation laws, finite elements, adaptive methods.

${ }^{1}$ Department of Mathematics, University of Crete, Heraklion 71409, Greece.

2 Department of Applied Mathematics, University of Crete, Heraklion 71409, Greece.

${ }^{3}$ Institute for Applied and Computational Mathematics, FORTH, Heraklion 71110, Greece.
} 
Conservation laws are related to the zero relaxation limits of appropriate models, cf. e.g., [8,9]. The relaxation model proposed by Jin and Xin [24] and used henceforth for designing schemes is

$$
\begin{aligned}
& u_{t}+v_{x}=0, \\
& v_{t}+c^{2} u_{x}=-\frac{1}{\varepsilon}(v-f(u)) .
\end{aligned}
$$

The system (1.3) can be viewed as a regularization of (1.2) by the wave operator,

$$
u_{t}+f(u)_{x}=-\varepsilon\left(u_{t t}-c^{2} u_{x x}\right) .
$$

In the zero relaxation limit $\varepsilon \rightarrow 0$, we recover (1.2) provided the subcharacteristic condition [34], holds: $-c<$ $f^{\prime}(u)<c,[24,29,37,38]$. The relaxation schemes proposed in [24] are based on the finite difference discretization of (1.3) treating the linear part in the usual upwind manner. The resulting relaxation schemes are used in the regime $\varepsilon \ll h$ or even when $\varepsilon \rightarrow 0$ (relaxed schemes). Here $h$ stands for the space discretization parameter. In this case therefore the induced regularization mechanism is still the artificial diffusion, see Section 2 for details.

Our approach has different motivation. Assume first for simplicity that the data of our problem and its solution in $[0, T]$ has compact support in a set $\Omega_{1} \subset \mathbb{R}$. Let $\Omega$ be an appropriate set such that $\Omega_{1} \subset \Omega$ where our numerical approximation will be sought. Then we consider a classical one dimensional $C^{0}$ finite element space defined on an element decomposition $\mathcal{T}_{h}$ of $\Omega$ :

$$
S_{h}=\left\{\phi \in C(\mathbb{R}):\left.\phi\right|_{K} \in \mathbb{P}_{p}(K), K \in \mathcal{T}_{h},\left.\phi\right|_{\Omega^{c}} \equiv 0\right\} .
$$

We denote by $\mathbb{P}_{q}(Q)$ the space of polynomials on $Q$ of degree at most $q$, by $K$ the elements (intervals in the one dimensional case) of the partition $\mathcal{T}_{h}$, and by $h_{K}$ the diameter of the element $K$. We then consider the finite element discretization of (1.3)

$$
\begin{aligned}
& \left(\partial_{t} u_{h}, \phi\right)+\left(\partial_{x} v_{h}, \phi\right)=0 \quad \forall \phi \in S_{h}, \\
& \left(\partial_{t} v_{h}, \psi\right)+c^{2}\left(\partial_{x} u_{h}, \psi\right)=-\frac{1}{\varepsilon}\left(v_{h}-f\left(u_{h}\right), \psi\right), \quad \forall \psi \in S_{h},
\end{aligned}
$$

where the unknowns $u_{h}, v_{h}$ are sought in $S_{h}$. The idea is to use (1.5) with very small $\varepsilon$. Since no extra diffusion mechanism is present in this scheme the regularization mechanism will be just the relaxation (or equivalently this of (1.4)). We will see in the sequel that the local mesh size close to the shock should be $h_{K} \leq c \varepsilon$. This requirement is compatible with the finite element approach based on unstructured meshes and mesh refinement. Indeed using appropriate estimators that locate the shock, (1.5) is combined with an adaptive method for the selection of the mesh yielding fine mesh in the shock regions and coarser mesh in the regular branches of the solution. The schemes constructed using this idea are based on a regularization mechanism with finite speed of propagation, do not need the solution of approximate local Riemann problems, can be formulated as low order or high order schemes, or even a combination of them $(h-p$ methods), and can be extended in multidimensions by using the finite element framework. On the other hand the difficulties of the problem are faced in the right choice of the mesh refinement mechanism and in the case of systems in the resolution of special structures such as contact discontinuities that need special treatment in the pde level (relaxation model), cf. [17]. One of the main characteristics of the finite element relaxation schemes proposed in the present paper is that are Riemann solvers free finite element schemes and thus they combine high accuracy and simplicity. Are related therefore to the central difference schemes of [39], see also the recent paper [31].

In the present paper we formulate semidiscrete Finite Element Relaxation Schemes in Section 2. We discuss then their consistency. Depending on the degree of the polynomials used and the regularity of the exact solution, the local truncation error is of optimal order. The behavior in shock regions is also discussed in addition to their relation to the classical finite difference relaxation schemes in the case of piecewise linear polynomials $(p=1)$. Although the focus of this paper is the study of our schemes in the one dimensional case $(N=1)$, in Section 2 we 
formulate as well schemes in the multidimensional case. In Section 3 we consider implicit-explicit fully discrete schemes. Treating the stiff nonlinear term implicitly [33], and the principal linear part explicitly (e.g., by the TVB schemes of $[45,46]$ ) we end up in a fully explicit scheme. Adaptivity and mesh refinement are considered in Section 4. Motivated by the results in $[19,20]$ we propose an error estimator that is able to accurately locate the shocks. Based on this information we can propose adaptive algorithms. Our choice in the computations is the algorithm designed on a given number of grid points. The algorithm selects then the location of the points based on the information provided by the estimator. Of course other methods are possible, see e.g., [20]. In Section 5 we discuss possible extensions of the schemes, as, e.g., methods with variable characteristic speed and variable relaxation parameter. Finally in Section 6 we present the computational performance of the method in test problems. We have selected Riemann problems and problems with smooth data. In Section 6.1 we consider the scalar one dimensional conservation law and in Section 6.2 the system of one dimensional elastodynamics.

Finite element methods for Hyperbolic Conservation Laws have been proposed and analyzed in the past, e.g., by Johnson and Szepessy $[25,26,45,49]$, Cockburn and Shou $[13,15,16]$, see the survey articles by Cockburn and Johnson in [14]. In the streamline diffusion method of [25,49] artificial diffusion has been added in the direction of streamlines along with special shock capturing terms. Finite element methods with discontinuous elements have been proposed in [23] and [16]. In particular in these papers the linking between elements is enforced by using appropriate one dimensional first order fluxes. These methods use polynomials of arbitrary degree and are formally of high order. The time discretization in [16] is done by using the TVB Runge-Kutta (RK) schemes of $[45,46]$. The same RK methods combined with appropriate diagonally implicit methods (DIRK) are used for the time discretization in the present paper. Adaptive finite element methods based on a posteriori estimates have been considered in [26] for the $\varepsilon$-viscous perturbation of one-dimensional systems of conservation laws. An alternative to finite elements is provided by the finite volume methods on unstructured grids. Among the numerous contributions in this direction, cf. e.g., $[10,14]$ and their references. Finite difference relaxation schemes were also considered in $[1,17,21,28,29]$. These methods can be viewed as discrete kinetic schemes, see the survey article of Perthame [41], see also [2,6]. Relaxation schemes on unstructured grids in multidimensions for scalar CL were introduced and analyzed in [27]. Methods based on adaptive grids for Conservation Laws were previously considered in $[5,26,35,47]$.

\section{Formulation of FERS}

\subsection{One dimensional systems}

Consider the system (1.1) with $N=1$. Then the relaxation system of [24]

$$
\begin{aligned}
& u_{t}+v_{x}=0, \\
& v_{t}+A u_{x}=-\frac{1}{\varepsilon}(v-F(u)),
\end{aligned}
$$

with $A=\operatorname{diag}\left\{a_{1}, a_{2}, \ldots a_{n}\right\}$ an appropriate diagonal matrix, corresponds to the regularization of (1.1) by introducing a wave operator to each component of the system. Then the corresponding finite element scheme is (with the same notation as in Sect. 1): Find $u_{h}, v_{h}$ in $S_{h}^{M}:=S_{h} \times \cdots \times S_{h}$ such that

$$
\begin{aligned}
& \left(\partial_{t} u_{h}, \phi\right)+\left(\partial_{x} v_{h}, \phi\right)=0 \quad \forall \phi \in S_{h}^{M}, \\
& \left(\partial_{t} v_{h}, \psi\right)+A\left(\partial_{x} u_{h}, \psi\right)=-\frac{1}{\varepsilon}\left(\left(v_{h}-F\left(u_{h}\right)\right), \psi\right), \quad \forall \psi \in S_{h}^{M} .
\end{aligned}
$$

For simplicity we take here $u_{h} \in S_{h}^{M}$ with $S_{h}$ defined in Section 1. Some systems may require to select $u_{h} \in \mathcal{S}_{h}$ where $\mathcal{S}_{h}=S_{h}^{(1)} \times \cdots \times S_{h}^{(M)}$, i.e., in general different meshes can be used for different components of $\mathcal{S}_{h}$. In order to ensure stability as in the scalar case, the matrix $A$ should be chosen in such a way that the following subcharacteristic condition holds, e.g., in the case of symmetric systems $A-F^{\prime}(u)^{2} \geq \mu I, \mu>0$. 
In case where the system admits a strictly convex entropy $\eta$, then $A$ should be chosen such that, $c f$. [50,51], $\frac{1}{2}\left(A^{T} \eta_{u u}(u)+\eta_{u u}(u) A\right)-\alpha{F^{\prime}}^{T}(u) F^{\prime}(u) \geq \mu I$ where $\eta_{u u}(u) \leq \alpha I$ and $\eta_{u u}$ denotes the Hessian of $\eta$. See also $[2,6]$ for stability conditions for (2.1).

The scheme (2.2) can be written in the form

$$
\begin{aligned}
& \left(\partial_{t} u_{h}, \phi\right)+\left(\partial_{x} v_{h}, \phi\right)=0 \quad \forall \phi \in S_{h}^{M}, \\
& \varepsilon\left(\partial_{t} v_{h}, \psi\right)+\varepsilon A\left(\partial_{x} u_{h}, \psi\right)+\left(\left(v_{h}-F\left(u_{h}\right)\right), \psi\right)=0, \quad \forall \psi \in S_{h}^{M},
\end{aligned}
$$

or (with the obvious notation)

$$
E_{h}\left(u_{h}, \phi ; v_{h}, \psi\right)=0, \quad \forall \phi, \psi \in S_{h}^{M} .
$$

As noted before (2.1) and hence (2.2) corresponds to the regularization of (1.1) by introducing a wave operator to each component of the system. This is probably not always the method of choice, in particular when the system admits linearly degenerate fields and contact discontinuities as in the case of Euler equations of gas dynamics. This is partly due to the fact that "exact" solvers for contact discontinuities that can work with relaxation are not available. In this case as suggested by Coquel and Perthame [17] we may introduce relaxation only to some of the components of the system and treat the other equations in the classical upwind manner. A relaxation model and a corresponding relaxation scheme for the equations of elastodynamics that introduces relaxation in one equation of the system was introduced in Gosse and Tzavaras [21]. This scheme was shown to be less diffusive than the relaxation scheme of [24]. One may formulate finite element relaxation schemes for the relaxation models of e.g., [17] and [21] (with appropriate modifications) and also try to find alternative approaches to the problem of resolution of the contact discontinuities. This will be the subject of a forthcoming investigation.

Consistency. To discuss the consistency properties of (2.1) we follow the classical finite element approach. Assume that the exact solution $u$ of (1.1) is sufficiently smooth in a domain $\Omega_{S}$. Let $v=F(u)$ and $\Pi$ be the finite element interpolant into $S_{h}[7]$. We have the following:

Proposition 2.1 Let $\phi, \psi \in S_{h}^{M}$ with compact support in $\Omega_{S}$ then under the above assumptions

$$
\left|E_{h}(\Pi u, \phi ; \Pi v, \psi)\right| \leq C(u)\left(h^{p}+\varepsilon\right)(\|\phi\|+\|\psi\|),
$$

and

$$
\left|E_{h}(\Pi u, \phi ; \Pi v, \psi)\right| \leq C(u)\left(h^{p+1}+\varepsilon\right)\left(\left\|\phi_{x}\right\|+\left\|\psi_{x}\right\|\right),
$$

where $E_{h}$ is defined in $(2.3)$ and $\|\cdot\|$ denotes the $L^{2}\left(\Omega_{S}\right)$ norm.

Proof. The finite element interpolant onto $S_{h}$ satisfies [7]

$$
\|w-\Pi w\|_{j, \Omega_{S}} \leq C h^{p+j}\|w\|_{p+1, \Omega_{S}}, \quad j=0,1,
$$

where $\|w\|_{m, \Omega_{S}}$ denotes the Sobolev norm of order $m$. For $v=f(u), u$ being the (smooth) solution of (1.1) we have

$$
\begin{aligned}
\left(\partial_{t} \Pi u, \phi\right)+\left(\partial_{x} \Pi v, \phi\right) & =\left(\partial_{t} u, \phi\right)+\left(\partial_{x} f(u), \phi\right)+\left(\partial_{t}(\Pi u-u), \phi\right)+\left(\partial_{x}(\Pi v-v), \phi\right) \\
& =\left(\partial_{t}(\Pi u-u), \phi\right)+\left(\partial_{x}(\Pi v-v), \phi\right) .
\end{aligned}
$$

Since $\Pi$ is independent of $t, \partial_{t}(\Pi u-u)=(\Pi-I) \partial_{t} u$. Also since $\phi$, has compact support in $\Omega_{S}$ we can integrate by parts the term

$$
\left(\partial_{x}(\Pi v-v), \phi\right) .
$$


Using the approximation properties of $\Pi$ we get the desired result for the first component of $E_{h}$. Similarly, for the second component,

$$
\begin{aligned}
\varepsilon\left(\partial_{t} \Pi v, \psi\right)+\varepsilon A\left(\partial_{x} \Pi u, \psi\right)+((\Pi F(u)-F(\Pi u)), \psi)= & \varepsilon\left(\partial_{t} v, \psi\right)+\varepsilon A\left(\partial_{x} u, \psi\right) \\
& +\varepsilon\left(\partial_{t}(\Pi v-v), \psi\right)+\varepsilon A\left(\partial_{x}(\Pi u-u), \psi\right) \\
& +((\Pi F(u)-F(u)), \psi)+((F(u)-F(\Pi u)), \psi)
\end{aligned}
$$

and the desired result follows as before.

These bounds are optimal, even for the corresponding linear problem. Note here that $\varepsilon$ is a fixed parameter that we choose according to the desired accuracy and at this level it is independent of the mesh size $h$. The estimates $(2.4 \mathrm{a}, \mathrm{b})$ combined with appropriate stability analysis can yield convergence rates for the scheme in the smooth case.

In order to get an idea of the behavior of the scheme close to the shock regions, we consider the case of a (locally) uniform mesh with size $h$. Then the finite element scheme for piecewise linear elements reduces to the central scheme

$$
\begin{aligned}
& \partial_{t} u_{j}+\frac{1}{2 h}\left[v_{j+1}-v_{j-1}\right]=0 \\
& \partial_{t} v_{j}+\frac{1}{2 h} c^{2}\left[u_{j+1}-u_{j-1}\right]=-\frac{1}{\varepsilon}\left[v_{j}-f\left(u_{j}\right)\right]
\end{aligned}
$$

Then $\left\{u_{j}\right\}$ satisfies,

$$
\partial_{t} u_{j}+\frac{1}{2 h}\left[f\left(u_{j+1}\right)-f\left(u_{j-1}\right)\right]+\varepsilon\left[\partial_{t t} u_{j}-c^{2} \frac{1}{4 h^{2}}\left[u_{j+2}-2 u_{j}+u_{j-2}\right]\right]=0 .
$$

This scheme is a third order approximation of the equation

$$
\partial_{t} u+f(u)_{x}+\varepsilon\left[\partial_{t t} u-c^{2} \partial_{x x} u\right]+\frac{h^{2}}{6} f(u)_{x x x}=0
$$

i.e., as expected the finite element discretization induces a dispersion term which is linear in the flux variable. Approximating one step further by using the Chapman-Enskog expansion we conclude

$$
\partial_{t} u+f(u)_{x}-\varepsilon \partial_{x}\left(\left(c^{2}-f^{\prime}(u)^{2}\right) \partial_{x} u\right)+\frac{h^{2}}{6} f(u)_{x x x}=0
$$

The limit dynamics of a dispersion-diffusion approximation of the Conservation Law is a subtle issue discussed, e.g., in [22]. Jacobs, McKinney and Shearer [22] showed that the equation

$$
\partial_{t} u^{\delta}+f\left(u^{\delta}\right)_{x}-\delta \partial_{x x} u^{\delta}+\beta \delta^{2} u_{x x x}^{\delta}=0,
$$

may have solutions that converge to nonclassical shocks as $\delta \rightarrow 0$. In addition even if this is not the case if the dispersion term is dominant the approximate solution will have oscillatory character close to the shock. Hence in order to exclude this possibility for our scheme the diffusion term should be dominant. This will enforce a condition of the form

$$
h<\gamma \varepsilon
$$

Note that $h$ in (2.7) is the local mesh size close to the shock and therefore in the mesh refinement strategy (2.7) should be respected only in a neighbourhood of the shock. The above arguments are supported by the numerical experiments in Section 6 . 
Let us now compare the above schemes with the finite difference relaxation schemes of [24]. The first order scheme in $[24]$ is

$$
\begin{aligned}
& \partial_{t} u_{j}+\frac{1}{2 h}\left[v_{j+1}-v_{j-1}\right]-\frac{1}{h} c\left[u_{j+1}-2 u_{j}+u_{j-1}\right]=0 \\
& \partial_{t} v_{j}+\frac{1}{2 h} c^{2}\left[u_{j+1}-u_{j-1}\right]-\frac{1}{h} c\left[v_{j+1}-2 v_{j}+v_{j-1}\right]=-\frac{1}{\varepsilon}\left[v_{j}-f\left(u_{j}\right)\right] .
\end{aligned}
$$

The model equation of this scheme is

$$
\begin{aligned}
& \partial_{t} u+v_{x}-h c u_{x x}=0, \\
& \partial_{t} v+c^{2} u_{x}-h c v_{x x}=-\frac{1}{\varepsilon}[v-f(u)] .
\end{aligned}
$$

Hence neglecting terms of higher order in terms of $h$ and $\varepsilon$ we conclude

$$
\partial_{t} u+f(u)_{x}+\varepsilon\left[\partial_{t t} u-c^{2} \partial_{x x} u\right]-h c u_{x x}=0
$$

In the classical relaxation schemes the idea is to compute in the regime $\varepsilon \ll h$, [24], therefore dominant term is the diffusion. Let us note also that if we compute in the regime $\varepsilon \ll h$ the approximate solution will be close to the solution of

$$
\partial_{t} u_{j}+\frac{1}{2 h}\left[f\left(u_{j+1}\right)-f\left(u_{j-1}\right)\right]-\frac{1}{2 h} c\left[u_{j+1}-2 u_{j}+u_{j-1}\right]=0
$$

which is a semidiscrete Lax-Friedrichs type scheme. Similar observations apply to high-order version of (2.8). It is to be noted that the relaxation schemes in [24] are very closely connected with the central schemes of [39], and especially with the semidiscrete central schemes suggested recently in [31], see also Section 5.

\subsection{Multidimensional systems}

The extension of the schemes in the multimensional case is straightforward by using the finite element framework. For this let $\mathcal{T}_{h}$ be a finite element decomposition of $\mathbb{R}^{N}$ into nonoverlapping, nonempty and open polyhedra. Let $h_{K}$ be the diameter of the polyhedron $K$ and let $h=\sup _{K \in \mathcal{T}_{h}} h_{K}<1$. We let

$$
S_{h}=\left\{\chi \in H_{0}^{1}(\Omega)^{M}:\left.\chi\right|_{K} \in \mathbb{P}_{p}(K)^{M}, K \in \mathcal{T}_{h}\right\}
$$

where $\Omega$ is an appropriate polyhedral domain outside of which our solution will be zero. The finite element relaxation schemes are defined by seeking $\left(u_{h}, v_{h, 1}, \ldots, v_{h, N}\right) \in S_{h}^{1+N}$ such that

$$
\begin{aligned}
& \left(\partial_{t} u_{h}, \phi\right)+\sum_{j=1}^{N}\left(\partial_{x_{i}} v_{h, i}, \phi\right)=0 \quad \forall \phi \in S_{h}, \\
& \left(\partial_{t} v_{h, i}, \psi\right)+\left(A_{i} \partial_{x_{i}} u_{h}, \psi\right)=-\frac{1}{\varepsilon}\left(\left(v_{h, i}-F_{i}\left(u_{h}\right)\right), \psi\right), \quad \forall \psi \in S_{h}, i=1, \ldots, N .
\end{aligned}
$$

Where the matrices $A_{i}$ should be chosen in order to satisfy certain subcharacteristic conditions. One may chose $A_{i}$ as in [24]. In analogy to (2.3) we write the multidimensional scheme as

$$
E_{h}\left(u_{h}, \phi ; v_{h}, \psi\right)=0, \quad \forall \phi, \psi \in S_{h}
$$


and then for $\Pi$ denoting the finite element interpolant into $S_{h}[7]$, we still have in smooth regions the consistency bounds

$$
\left|E_{h}(\Pi u, \phi ; \Pi v, \psi)\right| \leq C(u)\left(h^{p}+\varepsilon\right)(\|\phi\|+\|\psi\|),
$$

and

$$
\left|E_{h}(\Pi u, \phi ; \Pi v, \psi)\right| \leq C(u)\left(h^{p+1}+\varepsilon\right)\left(\|\phi\|_{1}+\|\psi\|_{1}\right)
$$

where $\|\cdot\|$ and $\|\cdot\|_{1}$ denote the $L^{2}$ and $H^{1}$ norms respectively.

In the multidimensional case the idea is still to use mesh refinement in the regions of singularity of the conservation law. In the multidimensional case however the selection of the mesh is crucial and needs careful analysis different than the one considered in the present paper in the case of one dimensional systems. Still the main advantage of $(2.9)$ is that the principal part of the operator is linear. We refer to $[36,42,47]$ where different approaches are proposed and analyzed for the resolution of the boundary layer for linear singularly perturbed problems with finite element meshes. Appropriate mesh refinement strategies for (2.9) will be discussed in a forthcoming work.

\section{Fully Discrete sChemes}

There are many alternatives for the time discretization of the semidiscrete problem (2.2), cf. e.g. the references cited in the introduction, where relevant methods for the time discretization of conservation laws are considered. In our case, it seems that Runge-Kutta methods are preferable due to the flexibility they allow which is suitable for adaptivity. A different choice would be a space time finite element treatment. We will present the scalar one dimensional case only, the extension to the other cases being obvious. We will consider therefore fully discrete schemes for the system

$$
\begin{aligned}
& \left(u_{h, t}, \varphi\right)+\left(v_{h, x}, \varphi\right)=0, \quad \forall \varphi \in S_{h} \\
& \left(v_{h, t}, \psi\right)+c^{2}\left(u_{h, x}, \psi\right)=-\frac{1}{\varepsilon}\left(v_{h}-f\left(u_{h}\right), \psi\right), \quad \forall \psi \in S_{h} .
\end{aligned}
$$

We discretize the system (3.1) in time using RK methods. We have to treat the stiff nonlinear term implicitly. The linear principal part of (3.1) can be treated explicitly [24,33]. Then as in [24] due to the structure of the system the resulting scheme will be linear. In order to decouple the system we proceed as follows: we discretize the first equation by using an Explicit RK (ERK) method while for the second we use an Diagonally Implicit RK (DIRK) method.

Let $\delta$ be the temporal stepsize (of course $\delta$ can be variable with $n$, but for simplicity we drop the subscript $n$ ) and let $t^{n}=t^{n-1}+\delta$. Assuming that the approximations at $t^{n},\left(u_{h}^{n}, v_{h}^{n}\right)$ have been computed then $\left(u_{h}^{n+1}, v_{h}^{n+1}\right)$ are defined by

$$
\begin{aligned}
& \left(u_{h}^{n+1}, \varphi\right)=\left(u_{h}^{n}, \varphi\right)+\delta \sum_{j=1}^{q} b_{j}\left\{-\left(v_{h, x}^{n, j}, \varphi\right)\right\} \\
& \left(v_{h}^{n+1}, \psi\right)=\left(v_{h}^{n}, \psi\right)+\delta \sum_{j=1}^{q} \tilde{b}_{j}\left\{-c^{2}\left(u_{h, x}^{n, j}, \psi\right)-\frac{1}{\varepsilon}\left(v_{h}^{n, j}-f\left(u_{h}^{n, j}\right), \psi\right)\right\}
\end{aligned}
$$


where the intermediate stages $\left(u_{h}^{n, j}, v_{h}^{n, j}\right)$ are given by the following coupled system of $q$-equations:

$$
\begin{aligned}
& \left(u_{h}^{n, i}, \varphi\right)=\left(u_{h}^{n}, \varphi\right)+\delta \sum_{j=1}^{i-1} a_{i j}\left\{-\left(v_{h, x}^{n, j}, \varphi\right)\right\}, \quad i=1, \ldots, q \\
& \left(v_{h}^{n, i}, \psi\right)=\left(v_{h}^{n}, \psi\right)+\delta \sum_{j=1}^{i} \tilde{a}_{i j}\left\{-c^{2}\left(u_{h, x}^{n, j}, \psi\right)-\frac{1}{\varepsilon}\left(v_{h}^{n, j}-f\left(u_{h}^{n, j}\right), \psi\right)\right\}, \quad i=1, \ldots, q
\end{aligned}
$$

and the set of constants $A=\left(a_{i j}\right), b=\left(b_{1}, \ldots, b_{q}\right), \tilde{A}=\left(\tilde{a}_{i j}\right), \tilde{b}=\left(\tilde{b}_{1}, \ldots, \tilde{b}_{q}\right), i, j=1, \ldots, q$ define the $q$-stage (ERK) and (DIRK) methods respectively.

In our experiments we use the following (ERK) methods proposed in $[45,46]$, which are of 2nd and 3rd order respectively

$$
A=\left(\begin{array}{ll}
0 & 0 \\
1 & 0
\end{array}\right), \quad b=\left(\begin{array}{c}
1 / 2 \\
1 / 2
\end{array}\right), \quad A=\left(\begin{array}{ccc}
0 & 0 & 0 \\
1 & 0 & 0 \\
1 / 4 & 1 / 4 & 0
\end{array}\right), \quad b=\left(\begin{array}{c}
1 / 6 \\
1 / 6 \\
2 / 3
\end{array}\right) .
$$

These explicit RK schemes are known to be appropriate for the discretization of conservation laws. The corresponding diagonally implicit methods (DIRK) that we use are

$$
A=\left(\begin{array}{cc}
0 & 0 \\
1 / 2 & 1 / 2
\end{array}\right) \quad b=\left(\begin{array}{l}
1 / 2 \\
1 / 2
\end{array}\right), \quad A=\left(\begin{array}{ccc}
0 & 0 & 0 \\
1 / 2 & 1 / 2 & 0 \\
1 / 4 & 0 & 1 / 4
\end{array}\right) \quad b=\left(\begin{array}{l}
1 / 6 \\
1 / 6 \\
2 / 3
\end{array}\right)
$$

The intermediate stages $\left(u_{h}^{n, j}, v_{h}^{n, j}\right), j=1, \ldots, q$ are evaluated at the same time levels $\tau=\tilde{\tau}=(0,1)$, and $\tau=\tilde{\tau}=(0,1,1 / 2)$ respectively. Note that evaluating the intermediate stages requires the solution of (3.3) which (since it can be decoupled) is a fully explicit finite element scheme and requires the inversion of a mass matrix. This can be further simplified by using appropriate numerical quadrature on the $L^{2}$ inner product, but we will not insist on that here.

It is to be noted that semidiscrete schemes of the previous section can be discretized in time with other methods too. In this direction let us mention the methods used recently in [31]. Fully implicit Runge Kutta schemes is also a choice it should be examined computationally.

\section{Mesh modification}

The method in the form used in this paper requires changing the finite element space with $n$. This is clear since, in our approach, when the shocks are moving the mesh should be chosen according the locations of shocks. Therefore for each discrete time node $t^{n}$ the approximation $u_{h}^{n}$ will be sought in $S_{h, n}$ where $n$ indicates that the space changes with $n$. For this let

$$
S_{h, n}=\left\{\phi \in C(\mathbb{R}):\left.\phi\right|_{K} \in \mathbb{P}_{p}(K), K \in \mathcal{T}_{h}^{n},\left.\phi\right|_{\Omega^{c}} \equiv 0\right\} .
$$

Where in the one dimensional case the elements will be intervals

$$
K=K_{j}^{n}=\left(x_{j-1}^{n}, x_{j}^{n}\right), \text { of the partition } \mathcal{T}_{h}^{n}=\left\{K_{j}^{n}, j=1, \ldots, N_{h}(n)\right\} .
$$

There are two alternatives in the implementation of (3.2), (3.3) since $u_{h}^{n}, v_{h}^{n} \in S_{h, n}$ and $u_{h}^{n+1}, v_{h}^{n+1} \in S_{h, n+1}$.

1. To replace $u_{h}^{n}, v_{h}^{n}$ in (3.2), (3.3) by their $L^{2}$ projections into $S_{h, n+1}$. In this case (3.2), (3.3) will not change.

2. To replace $u_{h}^{n}, v_{h}^{n}$ in (3.2), (3.3) by their finite element interpolants into $S_{h, n+1}$. In this case $u_{h}^{n}, v_{h}^{n}$ have to be replaced by $\Pi^{n+1} u_{h}^{n}, \Pi^{n+1} v_{h}^{n}$ in (3.2), (3.3). 


\section{Adaptive algorithms}

The methods (2.2) and (2.9) can be combined with adaptive algorithms for the selection of the mesh. This can be done with the help of a posteriori estimates. Such estimates are available, e.g., for finite difference schemes satisfying certain entropy inequalities [20] and for finite volume schemes [19,30], see also [11, 12, 26,39, 48]. In particular consider first order schemes written in the (viscous) form

$$
\left.v_{j}^{n+1}=v_{j}^{n}-\frac{\Delta t}{2 h_{j}}\left(f\left(v_{j+1}^{n}\right)-f\left(v_{j-1}^{n}\right)\right)\right)+\frac{\Delta t}{2 h_{j}}\left(Q_{j+\frac{1}{2}}^{n}\left(v_{j+1}^{n}-v_{j}^{n}\right)-Q_{j-\frac{1}{2}}^{n}\left(v_{j}^{n}-v_{j-1}^{n}\right)\right),
$$

where $S_{j}=\left(x_{j+\frac{1}{2}}, x_{j-\frac{1}{2}}\right), j \in \mathbb{Z}$, and $x_{j}$ denotes the middle point of $S_{j},\left|K_{j}\right|=h_{j} \leq h$. Then the piecewise constant numerical approximation $\left(v_{h},\left.v_{h}\right|_{S_{j} \times\left[t^{n}, t^{n+1}\right)}=v_{j}^{n}\right)$ generated by this scheme satisfies, under appropriate CFL conditions [20], the estimate

$$
\sup _{t \leq t^{n}}\left\|v_{h}(t)-u(t)\right\|_{L^{1}} \leq\left\|v_{h}(0)-u_{0}\right\|_{L^{1}}+C \sqrt{T V\left(u_{0}\right) M} \mathcal{E}\left(v_{h}, t^{n}\right),
$$

where

$$
\mathcal{E}\left(v_{h}, t^{n}\right)=\left\{\left(\sum_{m=0}^{n} \Delta t \sum_{j \in \mathbb{Z}} h_{j}\left|v_{j}^{m}-v_{j-1}^{m}\right|\right)^{1 / 2}+\sqrt{t^{n}} \max _{m \leq n}\left(\sum_{j \in \mathbb{Z}} h_{j}\left|v_{j}^{m}-v_{j-1}^{m}\right|\right)\right\} .
$$

In addition for high resolution schemes (including the SOR schemes of [40]) the following estimate was proved in $[20]$

$$
\sup _{t \leq t^{n}}\left\|v_{h}(t)-u(t)\right\|_{L^{1}} \leq\left\|v_{h}(0)-u_{0}\right\|_{L^{1}}+C \mathcal{E}\left(v_{h}, t^{n}\right)+C_{1} \mathcal{E}_{1}\left(v_{h}, t^{n}\right)
$$

where the new term $\mathcal{E}_{1}\left(v_{h}, t^{n}\right)$ measures the lack of entropy consistency of the scheme. It was shown in certain model cases that $\mathcal{E}_{1}\left(v_{h}, t^{n}\right)$ is of higher order compared to $\mathcal{E}\left(v_{h}, t^{n}\right)$. Thus the estimator $\mathcal{E}\left(v_{h}, t^{n}\right)$ controls the error for a wide class of schemes (similar observations hold for finite volume schemes, for the monotone case see $[19,30])$.

For systems of Conservation Laws there is no hope in general to prove such estimates. Nevertheless, motivated by the above discussion we can try to see if the estimator $\mathcal{E}$ can be proved useful, even if we do not have estimates like (4.2). Indeed, we can observe that for an approximation $u_{h}$ of the solution $u$ of the Conservation Law the summands of $\mathcal{E}\left(u_{h}\right)$,

$$
h_{j}\left|u_{h}\left(x_{j}, t^{m}\right)-u_{h}\left(x_{j-1} t^{m}\right)\right|
$$

locate accurately the (approximate) shock. This is also confirmed by the numerical experiments in Section 6 . The idea is to use this crucial information to design the adaptive algorithms.

To this end, for a given approximation $u_{h}^{n} \in S_{h, n}$ obtained by the scheme (3.2),(3.3), we define the estimator function $g$, to be the continuous piecewise linear function on the mesh of $S_{h, n}$ such that

$$
g\left(x_{i}^{n}\right)=g_{i}^{n}=h_{i}\left|u_{h}^{n}\left(x_{i}\right)-u_{h}^{n}\left(x_{i-1}\right)\right|
$$

More detailed choices for $g_{i}^{n}$ can be considered, using, e.g., differences at more points than only the end points of the interval, but for simplicity in the exposition we will work with (4.4). Figure 1 shows the form of $g$ for a given $u_{h}^{n}$.

Adaptive algorithm (Constant number of nodes). The following method is based on the principle that the number of nodes of the finite element space, $c f .(3.4)$, (3.5), at all times will be constant. This approach 

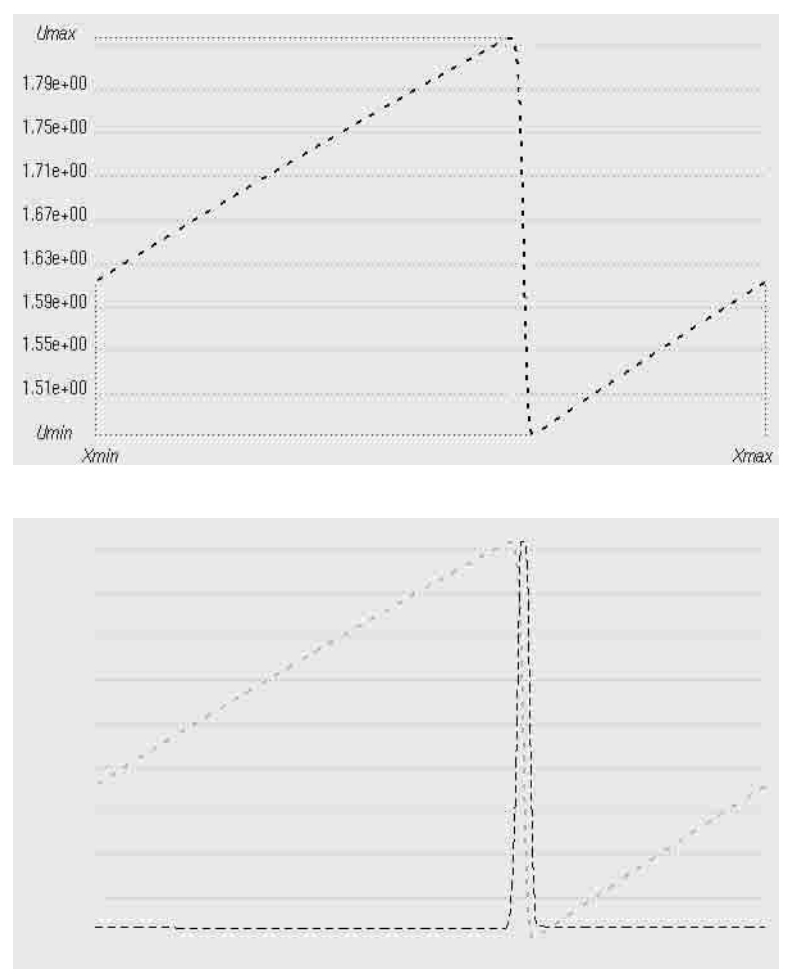

FIGURE 1. An approximate solution $u_{h}$ and its estimator function $g$.

although different is related to the "one shot" adaptive methods of $[3,4]$. (The resulting algorithm can be thus directly compared with traditional finite difference methods, at least in terms of the degree of freedoms used per time step.) In particular each approximation $u_{h}^{n}$ will be sought in $S_{h, n}$. This space changes with $n$, but the cardinality of $\mathcal{T}_{h}^{n}$ in its definition, (3.4), (3.5), is assumed to be a predescribed constant number $N_{h}$. Therefore the nodes of the partition $\mathcal{T}_{h}^{n}$ will be

$$
\left\{x_{0}^{n}, x_{1}^{n}, \ldots, x_{N_{h}}^{n}\right\}
$$

The problem is reduced to

given the nodes of $S_{h, n}$, choose the new set of nodes $\left\{x_{i}^{n+1}\right\}_{i=0}^{N_{h}}$ which defines $S_{h, n+1}$.

Our algorithm uses a normalization of the estimator function $g$ as density function of the new mesh. For this let $G$ be the average of $g$ in the computational domain $\Omega$,

$$
G(x)=\frac{g(x)}{\int_{\Omega} g(y) \mathrm{d} y} .
$$

Then $\int_{\Omega} G(x) \mathrm{d} x=1$ and $G$ will be used as a density function for the new mesh $\left\{x_{i}^{n+1}\right\}_{i=0}^{N_{h}}$. In particular the new mesh is defined in order

$$
\int_{x_{i}^{n+1}}^{x_{i+1}^{n+1}} G(x) \mathrm{d} x=\frac{1}{N_{h}}
$$


to be satisfied, i.e., $\left\{x_{i}^{n+1}\right\}_{i=0}^{N_{h}}$ equidistributes the mass of $G$. It is clear from the form of $G$ (its mass is concentrated at shocks) that this choice will distribute the points in such a way that many points will be placed in the shock areas.

Extensive computations that we performed indicate that what is important is the qualitative behavior of $G$, i.e., the computational solution is not very sensitive in variations of the mesh as soon as the following two criteria are met:

(1) there are enough points in the shock regions;

(2) the points reserved for the smooth regions are sufficient to resolve them accurately.

In this direction, in the computations we used as distribution function $G$ a modified function given if in (4.6) we replace $g$ by $g^{\alpha}, 0 \leq \alpha \leq 1$. For $\alpha=0, G$ will yield uniform mesh and for $\alpha=1$ the mesh given by (4.6). The introduction of this modification increases the flexibility for the selection of the mesh and influences the qualitative behavior of the previous procedure mainly in terms of the intensity of the refinement close to shocks.

\section{Extensions}

In this section we discuss possible extensions of the methods proposed.

\subsection{Variable characteristic speed}

It is clear that the subcharacteristic condition $(M=N=1)\left|f^{\prime}\left(u_{h}\right)\right|<c$ should be respected. On the other hand the factor $c^{2}-f^{\prime}\left(u_{h}\right)^{2}$ influences the artificial diffusion of the scheme, $c f$. the discussion after (2.6). This means that when $f^{\prime}\left(u_{h}\right)$ vary a lot it is possible that $c^{2}-f^{\prime}\left(u_{h}\right)^{2}$ to be much larger than it is necessary in some areas. Therefore, an alternative to this is to use $c$ variable with $u$. There are many different ways to do that. One is to use as $c$ a piecewise polynomial function such that in each cell $c$ dominates the local maximum of $\left|f^{\prime}\left(u_{h}\right)\right|$. This can be done also in an adaptive way in the fully discrete schemes without increasing the cost of the method in a significant way. Let us note here the strong link between the classical relaxation schemes with variable characteristic speed, $c f$. the discussion after (2.8), and the central schemes of [31].

\subsection{Variable relaxation parameter}

Since $\varepsilon$ influences both the regularization effect of the method and its accuracy in smooth regions, it might be useful for $\varepsilon$ to take different values in shock regions and different in smooth regions. It seems that in one dimensional problems this is not necessary (because we can take $\varepsilon$ very small) but probably in multidimensional problems this might be proven useful.

\section{Numerical EXAMPLES}

\subsection{Scalar equations}

We will consider first the Burgers equation,

$$
u_{t}+u u_{x}=0
$$

with initial value $u^{0}=0.75 \sin (\pi x)$ on $[0,2]$. In Figure 2 we can see the solutions with 200 nodes with uniform mesh and with mesh refinement at $t=0.52$ and $t=1$. The refinement provides an efficient mechanism that dumps completely the oscillations. The approximate solution with higher order polynomials $p=2,4$ is presented in Figure 3. The two solutions $(p=2,4)$ are indistinguishable. One can see also the grid selected by our adaptive code. For the second picture we consider a Riemann problem for a scalar equation with a nonconvex flux

$$
u_{t}+f(u)_{x}=0, \quad f(u)=u+0.3 u^{3} .
$$




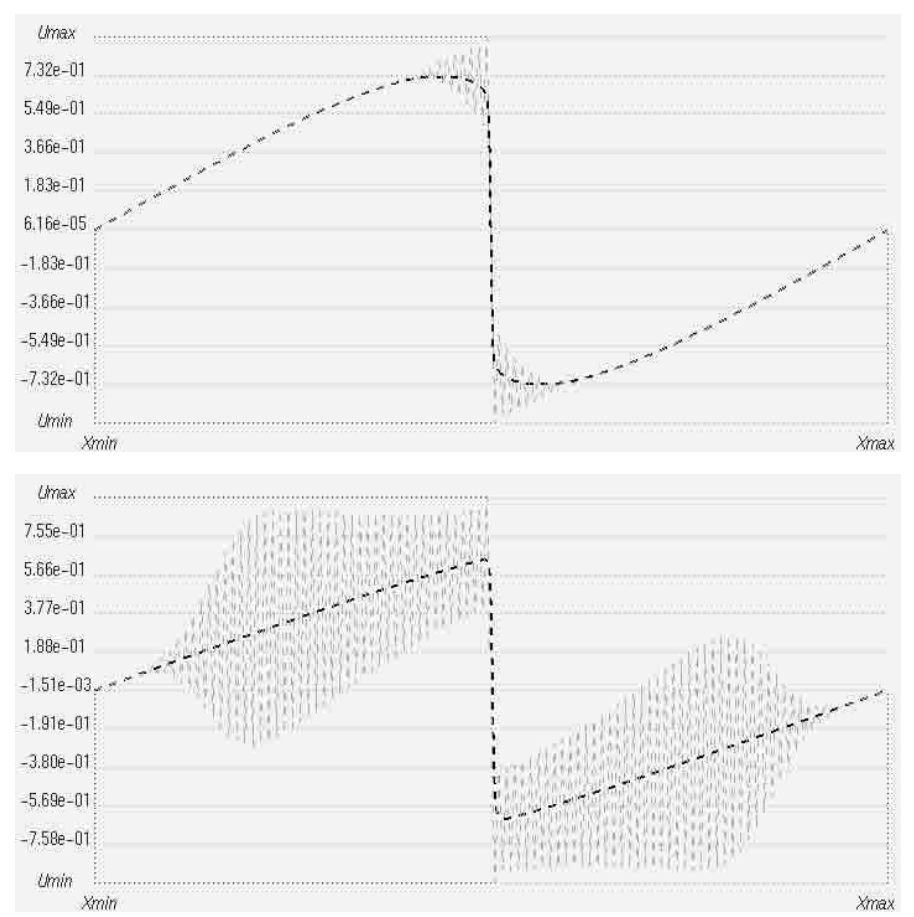

Figure 2. Two hundred nodes on $[0,2] p=1, T=0.52$ and $T=1 \varepsilon=5.0 e-06$, with refinement (black dotted line) and with uniform mesh (gray dotted line).
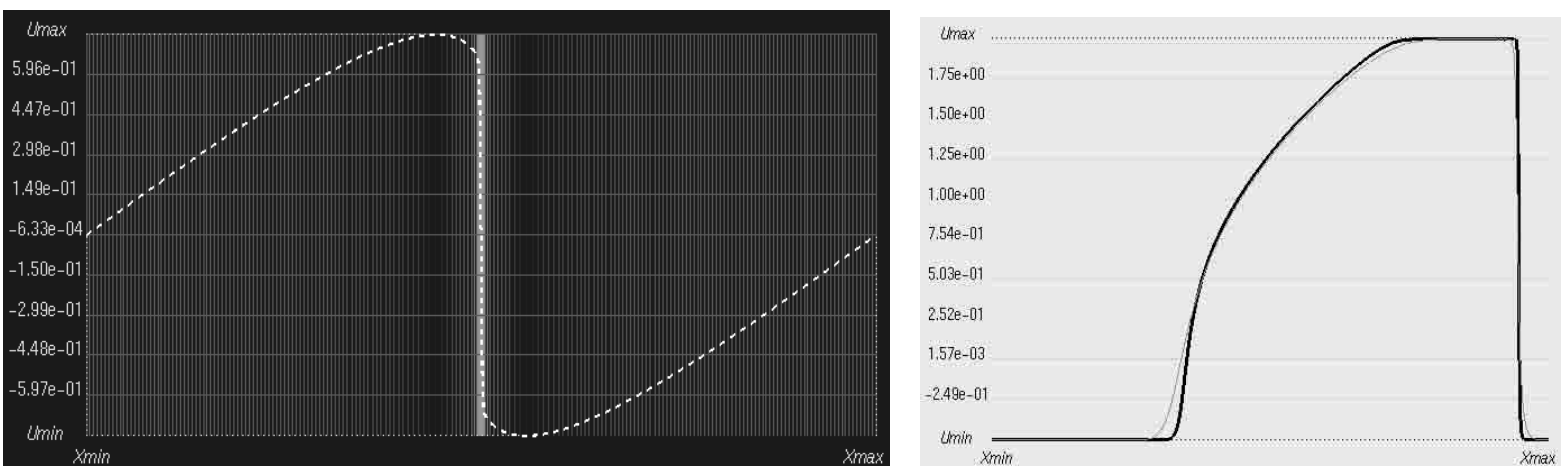

Figure 3. First picture: solutions and the grid with 200 nodes on $[0,2] p=1,4, T=0.52$. Second picture: (second example) 200 (gray line) and 400 nodes (black line) on $[-1,1] p=1$, $T=0.2 \varepsilon=3.0 e-07$, with refinement.

The initial value is $u^{0}=-1 / 2$ on $[-1,-1 / 2] \cup[1 / 2,1]$ and $u^{0}=2$ on $[-1 / 2,1 / 2]$. The solutions with 200 and 400 nodes are displayed. 

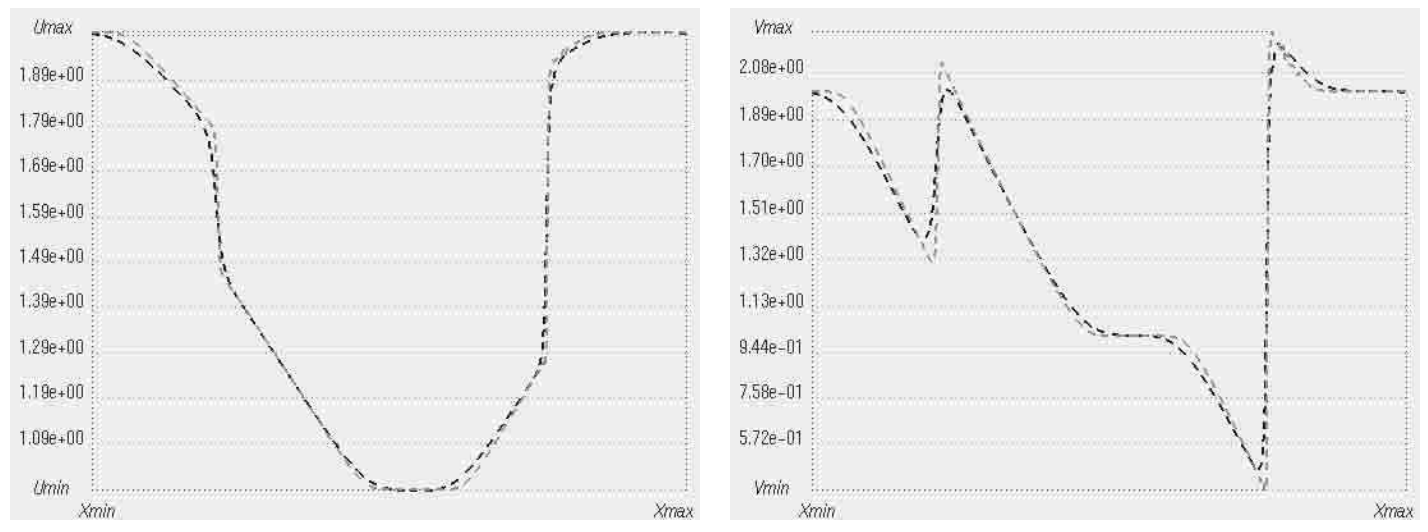

Figure 4. (System with Riemann data) 200 nodes on $[0,1] p=1, T=0.35 \varepsilon=5.0 e-07$, with refinement (black dotted lines) and $p=4, T=0.35 \varepsilon=5.0 e-06$, with refinement (gray dotted lines).

\subsection{System of elastodynamics}

We consider the one dimensional system of nonlinear elastodynamics

$$
\begin{aligned}
& u_{1, t}-u_{2, x}=0 \\
& u_{2, t}-f\left(u_{1}\right)_{x}=0 .
\end{aligned}
$$

The corresponding relaxation system considered here is

$$
\begin{aligned}
& u_{1, t}-v_{1, x}=0 \\
& u_{2, t}-v_{2, x}=0 \\
& v_{1, t}-a_{1} u_{1, x}=-\frac{1}{\varepsilon}\left(v_{1}-u_{2}\right) \\
& v_{2, t}-a_{2} u_{2, x}=-\frac{1}{\varepsilon}\left(v_{2}-f\left(u_{1}\right)\right) .
\end{aligned}
$$

Under certain hypotheses on $f$ and for $a_{1}, a_{2}$ sufficiently large a rigorous passage from (6.2) to (6.1) was established in [44].

We consider two test problems for this system with $f(v)=v+v^{3}$. First with Riemann data $u_{1}(0)=u_{2}(0)=2$ on $[0,1 / 4] \cup[3 / 4,1]$ and $u_{1}(0)=u_{2}(0)=1$ on $[1 / 4,3 / 4]$ see Figures 4 to 6 . The computations show that methods with piecewise linears and higher order polynomials give satisfactory results both in terms of accuracy and in terms of the qualitative behavior of the approximate solutions. For the same number of nodes high order $(p=2,3,4)$ methods give better resolution in shocks and also in smooth areas. In Figures 7 to 10 we present computations with smooth data $u_{1}(0)=u_{2}(0)=1+3 \sin (\pi x)$ on $[0,2]$. This is a non trivial problem from computational point of view, since the smooth parts of the solution have non trivial structure. There it is clear that high order methods have an advantage. In Figure 10 we can see a comparison of the computed solutions for two different values of the relaxation parameter $\varepsilon(\varepsilon=3.0 e-06$, and $\varepsilon=1.5 e-06$.)

Some general remarks for our computations are in order. In the above examples we did use constant characteristic speeds (for each run). One observation is that the characteristic speeds needed for successful runs vary with the order of the method. High order methods need larger values of $a_{1}, a_{2}$. In particular, in the case of the system with Riemann data (Fig. 5, 200 nodes) we have taken $a_{1}=a_{2}=15$ for $p=1$ and $a_{1}=a_{2}=250$ for $p=4$. In addition larger values for the characteristic speeds are needed if we have many nodes, e.g., in Figures 6 and 7 where we have 400 nodes we have taken $a_{1}=15, a_{2}=50$ for $p=1$ and $a_{1}=a_{2}=400$ for $p=2$. 

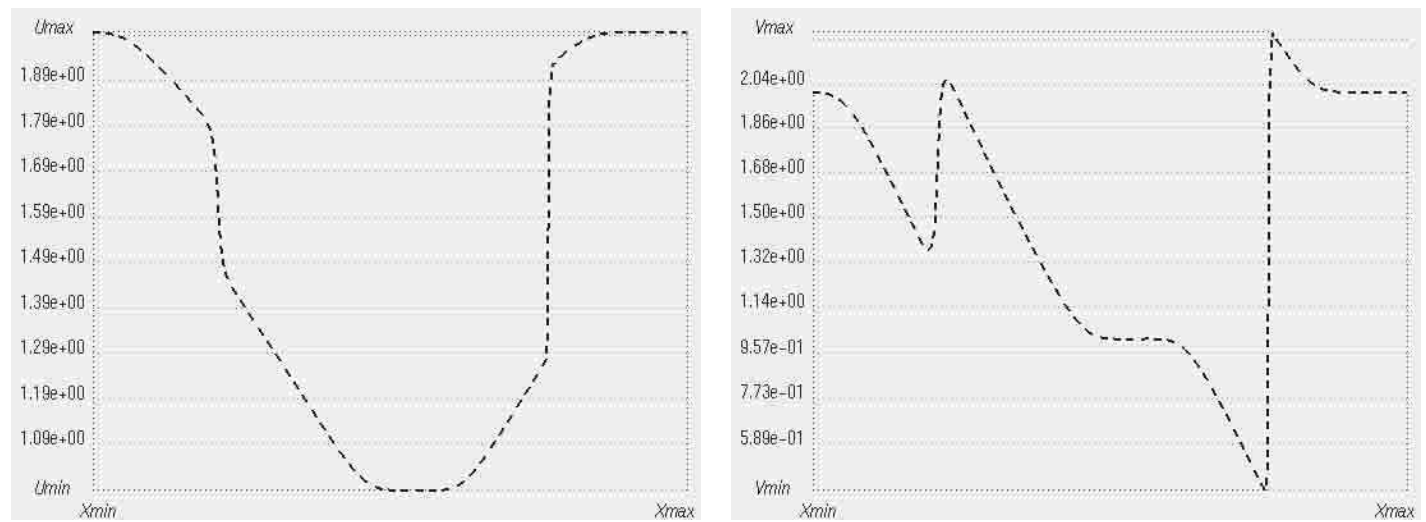

Figure 5. (System with Riemann data) 400 nodes on $[0,1] p=1, T=0.35 \varepsilon=5.0 e-07$.
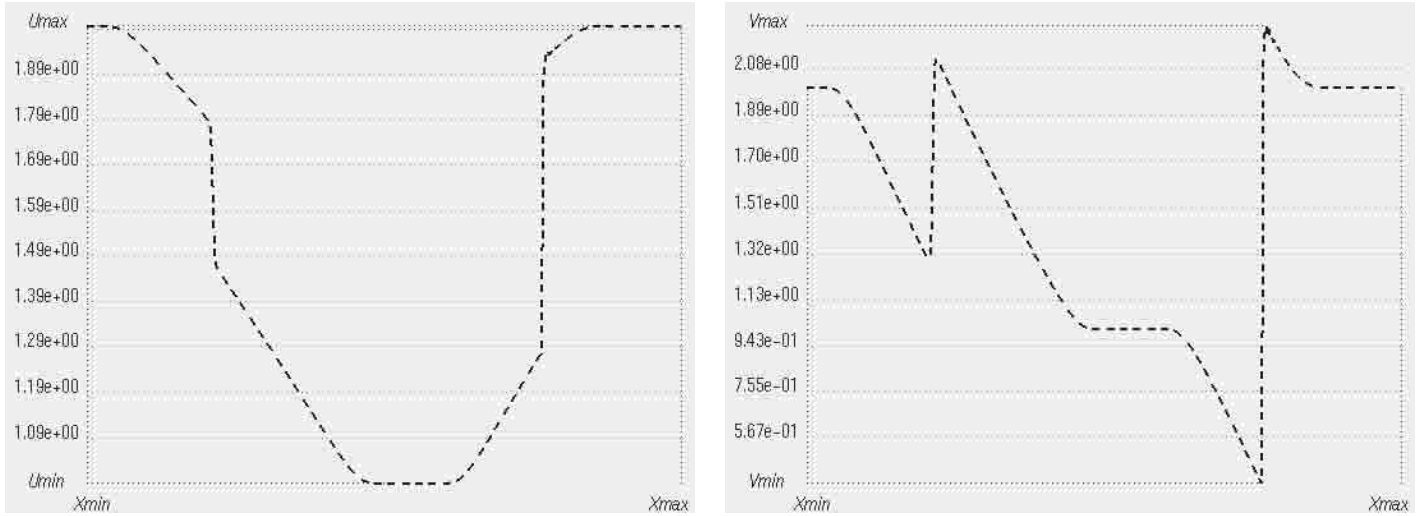

Figure 6. (System with Riemann data) 400 nodes on $[0,1], p=2, T=0.35 \varepsilon=5.0 e-07$.
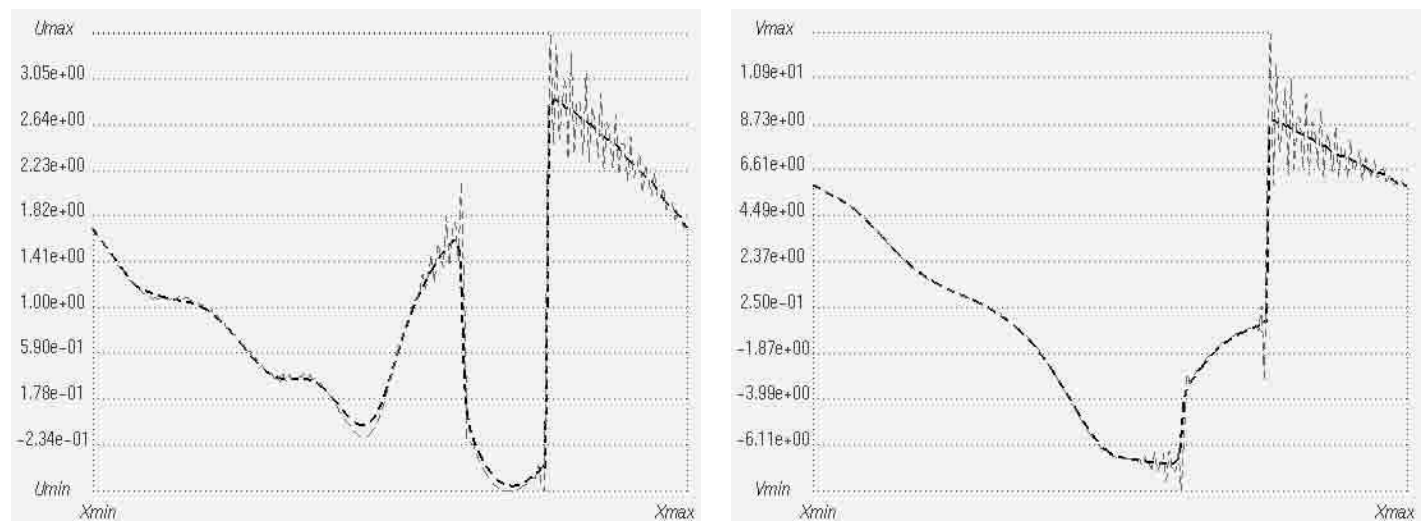

Figure 7. (System with smooth data) 200 nodes on $[0,2] p=1, T=0.2 \varepsilon=5.0 e-06$, with refinement (black dotted lines) and with uniform mesh (gray dotted lines). 

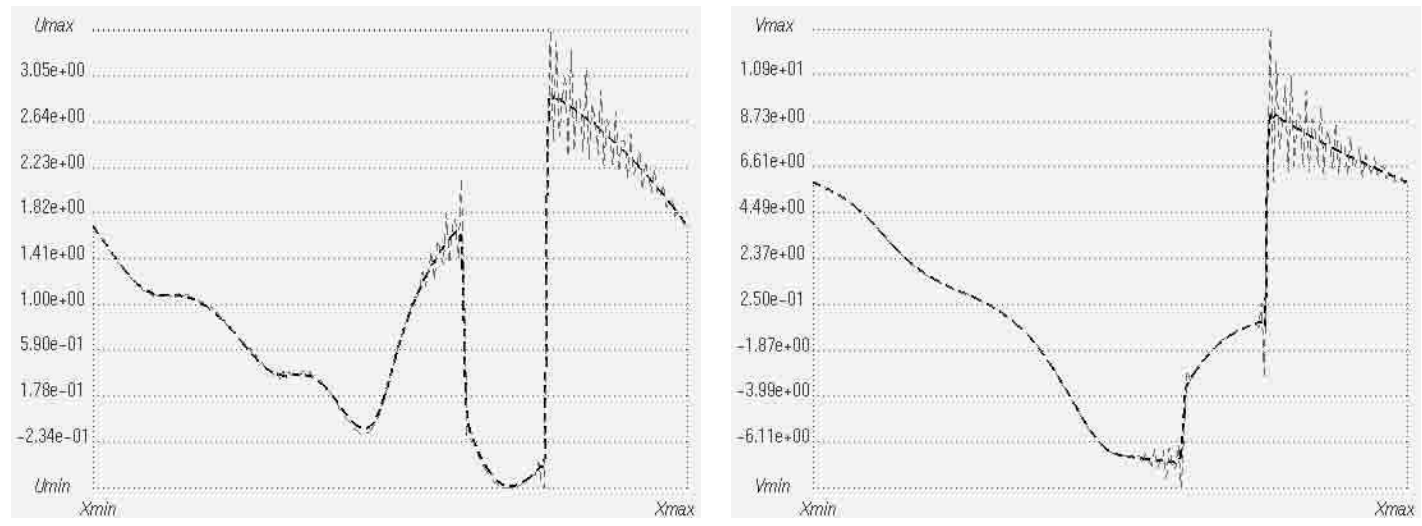

Figure 8. (System with smooth data) 200 nodes on $[0,2] p=2, T=0.2 \varepsilon=5.0 e-06$, with refinement (black dotted lines) and with uniform mesh for $p=1$ (gray dotted lines).
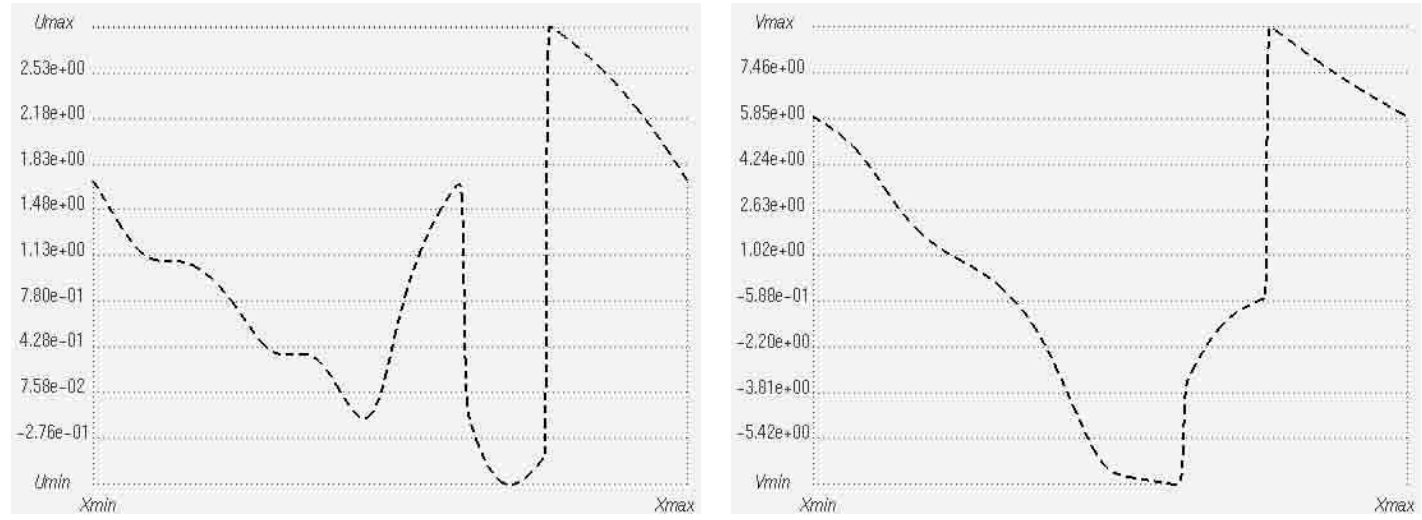

Figure 9. (System with smooth data) 400 nodes on $[0,2] p=2, T=0.2 \varepsilon=3.0 e-06$, with refinement.
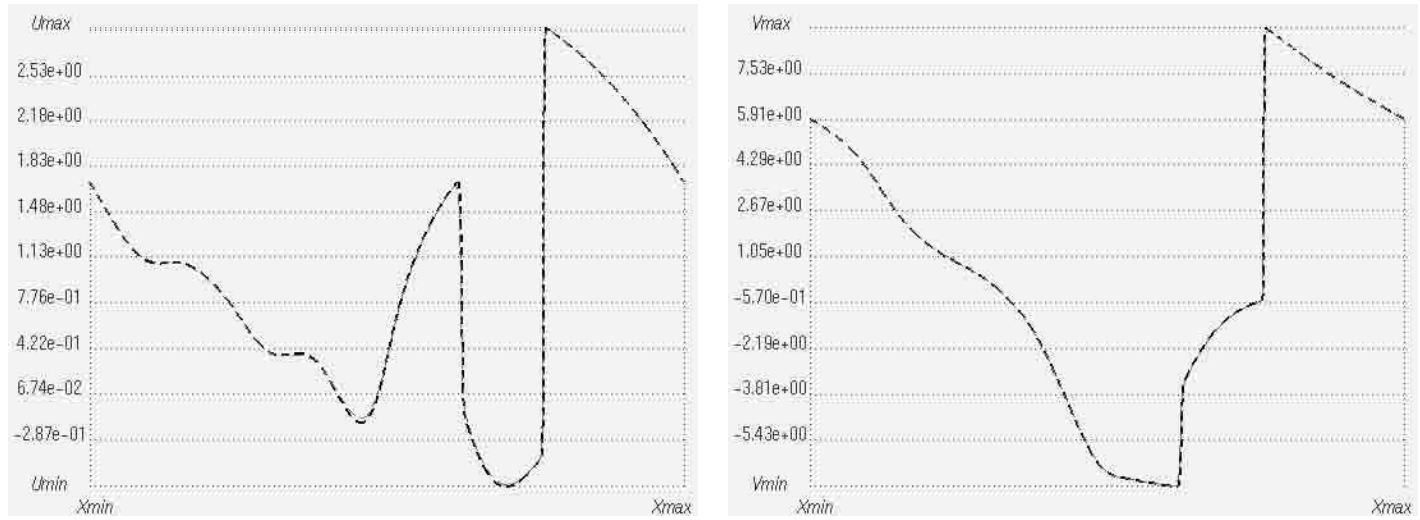

Figure 10. (System with smooth data) 400 nodes on $[0,2] p=3, T=0.2 \varepsilon=3.0 e-06$, and $\varepsilon=1.5 e-06$, with refinement. 
In general there is not restriction in the selection of the Runge-Kutta method with respect of the space accuracy. All combinations give satisfactory results. In the computations we have chosen higher order methods in time when higher than $p=1$ piecewise polynomials were used for the space discretization. The parameter $\varepsilon$ is fixed a priori to be very small and then we select the other parameters of the method. For the one dimensional examples considered here we were able to get very small $\varepsilon$, $c f$. also Section 5 . Finally (2.7) is respected by the selection of the mesh done by our adaptive algorithm. In particular the computations show that although $\varepsilon$ is very small, the minimum interval of the partition is much larger, i.e., $\gamma$ in $(2.7)$ is of the order of $10^{2}-10^{3}$.

Acknowledgements. The authors would like to thank A. Tzavaras for his interest in this work and many valuable discussions. This work is partially supported by European Union TMR grant No. FMRXCT960033 (HCL).

\section{REFERENCES}

[1] D. Aregba-Driollet and R. Natalini, Convergence of relaxation schemes for conservation laws. Appl. Anal. 61 (1996) 163-193.

[2] D. Aregba-Driollet and R. Natalini, Discrete kinetic schemes for multidimensional systems of conservation laws. SIAM J. Numer. Anal. 37 (2000) 1973-2004.

[3] I. Babuška, The adaptive finite element method. TICAM Forum Notes no 7, University of Texas at Austin (1997).

[4] I. Babuška and W. Gui, Basic principles of feedback and adaptive approaches in the finite element method. Comput. Methods Appl. Mech. Engrg. 55 (1986) 27-42.

[5] M. Berger and R. LeVeque, Adaptive mesh refinement using wave-propagation algorithms for hyperbolic systems. SIAM J. Numer. Anal. 35 (1998) 2298-2316.

[6] F. Bouchut, Construction of BGK models with a family of kinetic entropies for a given system of conservation laws. J. Statist. Phys. 95 (1999) 113-170.

[7] S.C. Brenner and L.R. Scott, The Mathematical Theory of Finite Element Methods. Springer-Verlag, New York (1994).

[8] R.E. Caflisch and G.C. Papanicolaou, The fluid dynamical limit of a nonlinear model Boltzmann equation. Comm. Pure Appl. Math. 32 (1979) 589-616.

[9] G.-Q. Chen, C.D. Levermore and T.-P. Liu, Hyperbolic conservation laws with stiff relaxation terms and entropy. Comm. Pure Appl. Math. 47 (1994) 789-830.

[10] B. Cockburn, F. Coquel and P. LeFloch, An error estimate for finite volume methods for conservation laws. Math. Comp. 64 (1994) $77-103$.

[11] B. Cockburn and H. Gau, A posteriori error estimates for general numerical methods for scalar conservation laws. Math. Appl. Comp. 14 (1995) 37-47.

[12] B. Cockburn and P.-A. Gremaud, Error estimates for finite element methods for scalar conservation laws. SIAM J. Numer. Anal. 33 (1996) 522-554.

[13] B. Cockburn, S. Hou and C.-W. Shu, The Runge-Kutta local projection discontinuous Galerkin finite element method for conservation laws. IV. The multidimensional case. Math. Comp. 54 (1990) 545-581.

[14] B. Cockburn, C. Johnson, C.-W. Shu and E. Tadmor, Advanced Numerical Approximation of Nonlinear Hyperbolic Equations. A. Quarteroni (Ed.), Lect. Notes Math. 1697, Springer-Verlag (1998).

[15] B. Cockburn, S.Y. Lin and C.-W. Shu, TVB Runge-Kutta local projection discontinuous Galerkin finite element method for conservation laws. III. One-dimensional systems. J. Comput. Phys. 84 (1989) 90-113.

[16] B. Cockburn and C.-W. Shu, TVB Runge-Kutta local projection discontinuous Galerkin finite element method for conservation laws. II. General framework. Math. Comp. 52 (1989) 411-435.

[17] F. Coquel and B. Perthame, Relaxation of energy and approximate Riemann solvers for general pressure laws in fluid dynamics. SIAM J. Numer. Anal. 35 (1998) 2223-2249.

[18] K. Dekker and J.D. Verwer, Stability of Runge-Kutta Methods for Stiff Nonlinear Differential Equations. CWI Monographs, North-Holland, Amsterdam (1984).

[19] L. Gosse and Ch. Makridakis, A-posteriori error estimates for numerical approximations to scalar conservation laws: schemes satisfying strong and weak entropy inequalities. IACM-FORTH Technical Report 98-4 (1998).

[20] L. Gosse and Ch. Makridakis, Two a posteriori error estimates for one dimensional scalar conservation laws. SIAM J. Numer. Anal. 38 (2000) 964-988.

[21] L. Gosse and A. Tzavaras, Convergence of relaxation schemes to the equations of elastodynamics. Math. Comp. (to appear).

[22] D. Jacobs, B. McKinney, M. Shearer, Travelling wave solutions of the modified Korteweg-de Vries-Burgers equation. J. Differential Equations 116 (1995) 448-467.

[23] J. Jaffré, C. Johnson and A. Szepessy, Convergence of the discontinuous Galerkin finite element method for hyperbolic conservation laws. Math. Models Methods Appl. Sci. 5 (1995) 367-386.

[24] S. Jin and Z. Xin, The relaxing schemes for systems of conservation laws in arbitrary space dimensions. Comm. Pure Appl. Math. 48 (1995) 235-277. 
[25] C. Johnson and A. Szepessy, On the convergence of a finite element method for a nonlinear hyperbolic conservation law. Math. Comp. 49 (1987) 427-444.

[26] C. Johnson and A. Szepessy, Adaptive finite element methods for conservation laws. Part I: The general approach. Comm. Pure Appl. Math. 48 (1995) 199-234.

[27] T. Katsaounis and Ch. Makridakis, Finite volume relaxation schemes for multidimensional conservation laws. Math. Comp. (to appear).

[28] M.A. Katsoulakis, G.T. Kossioris and Ch. Makridakis, Convergence and error estimates of relaxation schemes for multidimensional conservation laws. Comm. Partial Differential Equations 24 (1999) 395-424.

[29] M.A. Katsoulakis and A.E. Tzavaras, Contractive relaxation systems and the scalar multidimensional conservation law. Comm. Partial Differential Equations 22 (1997) 195-233.

[30] D. Kröner and M. Ohlberger, A posteriori error estimates for upwind finite volume schemes for nonlinear conservation laws in multidimensions. Math. Comp. 69 (2000) 25-39.

[31] A. Kurganov and E. Tadmor, New high resolution central schemes for nonlinear conservation laws and convection-diffusion equations. J. Comput. Phys. 160 (2000) 241-282

[32] N.N. Kuznetzov, Accuracy of some approximate methods for computing the weak solutions of a first-order quasi-linear equation. USSR Comput. Math. Math. Phys. 16 (1976) 105-119.

[33] R.J. LeVeque and H.C. Yee, A study of numerical methods for hyperbolic conservation laws with stiff terms. J. Comput. Phys. 86 (1990) 187-210.

[34] T.-P. Liu, Hyperbolic conservation laws with relaxation. Comm. Math. Phys. 108 (1987) 153-175.

[35] B.J. Lucier, A moving mesh numerical method for hyperbolic conservation laws. Math. Comp. 40 (1983) 91-106.

[36] J.J.H. Miller, E. O'Riordan and G.I. Shishkin, Fitted numerical methods for singular perturbation problems. Error estimates in the maximum norm for linear problems in one and two dimensions. World Scientific Publishing Co., River Edge, NJ (1996).

[37] R. Natalini, Convergence to equilibrium for the relaxation approximations of conservation laws. Comm. Pure Appl. Math. 8 (1996) 795-823.

[38] R. Natalini, A discrete kinetic approximation of entropy solutions to multidimensional scalar conservation laws. J. Differential Equations 148 (1998) 292-317.

[39] H. Nessyahu and E. Tadmor, Non-oscillatory central differencing for hyperbolic conservation laws. J. Comput. Phys. 87 (1990) 408-463.

[40] S. Osher and E. Tadmor, On the convergence of difference approximations to scalar conservation laws. Math. Comp. 50 (1988) $19-51$.

[41] B. Perthame, An introduction to kinetic schemes for gas dynamics, in: An introduction to recent developments in theory and numerics for conservation laws, D. Kröner, M. Ohlberger and C. Rohde (Eds.), Lect. Notes Comput. Sci. Eng. 5, SpringerVerlag (1998) 1-27.

[42] H.-G. Roos, M. Stynes and L. Tobiska, Numerical methods for singularly perturbed differential equations. Convection-diffusion and flow problems. Springer-Verlag, Berlin (1996).

[43] H.J. Schroll, A. Tveito and R. Winther, An $L^{1}$ error bound for a semi-implicit difference scheme applied to a stiff system of conservation laws. SIAM J. Numer. Anal. 34 (1997) 1152-1166.

[44] D. Serre, Relaxation semi linéaire et cinétique des systèmes de lois de conservation. Ann. Inst. H. Poincaré, Anal. Non Linéaire 17 (2000) 169-192.

[45] C.-W. Shu, Total-variation-diminishing time discretizations. SIAM J. Sci. Comput. 9 (1988) 1073-1084.

[46] C.-W. Shu and S. Osher, Efficient implementation of essentially nonoscillatory shock-capturing schemes. J. Comput. Phys. 77 (1988) 439-471.

[47] T. Sonar and E. Süli, A dual graph-norm refinement indicator for finite volume approximations of the Euler equations. Numer. Math. 78 (1998) 619-658.

[48] E. Süli, A-posteriori error analysis and adaptivity for finite element approximations of hyperbolic problems, in: An introduction to recent developments in theory and numerics for conservation laws, D. Kröner, M. Ohlberger and C. Rohde (Eds.), Lect. Notes Comput. Sci. Eng. 5, Springer-Verlag (1998) 123-194.

[49] A. Szepessy, Convergence of a shock-capturing streamline diffusion finite element method for a scalar conservation law in two space dimensions. Math. Comp. 53 (1989) 527-545.

[50] A. Tzavaras, Viscosity and relaxation approximation for hyperbolic systems of conservation laws, in: An introduction to recent developments in theory and numerics for conservation laws, D. Kröner, M. Ohlberger and C. Rohde (Eds.), Lect. Notes Comput. Sci. Eng. 5, Springer-Verlag (1998) 73-122.

[51] A. Tzavaras, Materials with internal variables and relaxation to conservation laws. Arch. Rational Mech. Anal. 146 (1999) 129-155. 